\title{
Методика реализации проекта автономного энергообеспечения в зависимости от стадий жизненного цикла
}

\section{Жильцов С.A.1}

${ }^{1}$ Российский университет дружбы народов, Москва, Россия

\section{АННОТАЦИЯ:}

Охарактеризовано возможное разнообразие и особенности проектов автономного энергоснабжения. Показано, что индивидуальные особенности каждого проекта автономного энергообеспечения требуют выделения стадий жизненного цикла проекта (ЖЦП) для его эффективной реализации. Выявлены базовые стадии ЖЦП автономного энергообеспечения. Предложена методика реализации проекта автономного энергообеспечения на различных стадиях ЖЦП.

КЛЮЧЕВЫЕ СЛОВА: энергообеспечение, автономные потребители, жизненный цикл проекта, фазы и стадии проекта, методика реализации проекта.

Method of implementation of the autonomous power supply project depending on the stages of the life cycle

\section{Zhiltsov S.A.}

${ }^{1}$ RUDN University, Russia

\section{Введение}

Сратегия развития удаленных территорий неразрывно связана с энергообеспечением (ЭО) автономных потребителей (АП), где невозможно или нецелесообразно возведение централизованных сетей энергоснабжения. Перспективным представляется использование возможностей повышения качества жизни и развития экономики удаленных территорий за счет реализации инновационных проектов [1] (Seliverstov, 2013) автономного энергоснабжения.

Известно, что инновационный проект, независимо от его назначения, характеризуется определенными стадиями жизненного цикла, при этом существующие научные исследования в сфере методологии управления проектами не отражают особенностей проектов ЭО АП. В этой связи, актуальными являются исследования, направленные на выявление стадий жизненного цикла проекта (ЖЦП) ЭО АП и особенностей управления данными проектами в зависимости от стадии ЖЦП. 
Целью данного исследования является формирование методики реализации проекта ЭО АП, в зависимости от стадий ЖЦП. Достижение цели производилось путем решения задач: обобщения особенностей проектов ЭО АП; выявления базовых стадий ЖЦП ЭО АП; разработки методики реализации проекта ЭО АП в зависимости от стадий ЖЦП.

\section{Особенности проектов автономного энергоснабжения}

Согласно Стратегии инновационного развития России, до 2020 г. и энергетической стратегии до 2030 г., перспективна разработка пилотных проектов «умных сетей» (smart grid), включающих цифровые технологии и распределенные источники энергии, в частности возобновляемые. Существуют внедрения ряда комплексных проектов и отдельные элементы smart grid на территории России, что, однако, не приводит к ожидаемому тиражированию и масштабированию проектов [2] (Kuznetsov, 2016) в связи с необходимостью дальнейшего решения проблем нормативно-правового регулирования, а также стандартизации и унификации оборудования, приборов учета и передаваемой информации [3].

Наиболее широко в проектах ЭО АП представлены дизельные и газовые станции, при этом последние могут иметь поршневое и турбинное устройство. Сравнительные исследования проектов централизованного и автономного ЭО с применением газотурбинных систем в экстремальных северных условиях на основе критериев чистой приведенной стоимости и минимальной цены на электроэнергию показали предпочтительность централизованного ЭО. Однако оценка чувствительности проекта показала высокую корреляцию стоимости проекта с потенциальным ростом платы и

\section{ABSTRACT:}

The possible diversity and features of autonomous power supply projects are characterized. It is shown that the individual features of each project of autonomous power supply require the identification of the stages of the project life cycle (PLC) for its effective implementation. The basic stages of PLC of autonomous power supply project are identified. A method of implementation of the autonomous power supply project at various stages of the PLC is proposed.

KEYWORDS: power supply, autonomous consumers, project life cycle, phases and stages of the project, method of project implementation

JEL Classification: Q40, Q43, Q48, Q49

Received: 22.09.2018 / Published: 30.12.2018

(C) Author(s) / Publication: CREATIVE ECONOMY Publishers

For correspondence: Zhiltsov S.A. (zhiltsovsaamail.ru)

\section{CITATION:}

Zhiltsov S.A. (2018) Metodika realizatsii proekta avtonomnogo energoobespecheniya $v$ zavisimosti ot stadiy zhiznennogo tsikla [Method of implementation of the autonomous power supply project depending on the stages of the life cycle]. Voprosy innovatsionnoy ekonomiki. 8. (4). - 731-740. doi: 10.18334/vinec.8.4.39433 
штрафа за сжигание газа, что делает предпочтительными автономные системы, кроме того, на ряде удаленных территорий, вариант автономного ЭО является единственно возможным с технической точки зрения [4] (Ershov, 2017).

Недостаточная распространенность возобновляемых источников энергии (ВИЭ) в проектах ЭО АП, несмотря на более низкую стоимость по сравнению с дизельными станциями и отсутствие необходимости сжигания природных ресурсов, обусловлена сезонностью и значительной нестабильностью ВИЭ. Устранение данного ограничения возможно при использовании проектов ЭО АП на основе гибридных систем, сочетающих первичный и дополнительный источники питания (ВИЭ), аварийный (топливный) источник, устройство хранения энергии, метеостанцию и контроллеры и пр. При этом состав гибридной системы может изменяться в зависимости от ее географического расположения, а основное достоинство таких систем заключается в автоматическом реагировании на нехватку энергии в различных сетевых узлах путем корректировки ее подачи внутри сети в зависимости от потребностей [5] (Fedak, Ulbrich, Anweiler, Jarosz, 2017).

Проекты гибридных микросетей для ЭО АП требуют предварительного моделирования эффективности энергоснабжения и технико-экономического обоснования. Например, для удаленных поселений на 100 чел., рекомендуется микросеть, включающая фотогальванический элемент, ветряную турбину, солнечную батарею, дизельгенератор и сопутствующие накопительные и распределительные устройства [6] (Fikari, 2015).

Исследования, направленные на оценку проектов различных комплектаций автономных гибридных систем, использующих солнечную энергию, например: (1) фотоэлектрических с дизельным генератором; (2) фотоэлектрических и аккумулятора; (3) фотоэлектрических с дизельным генератором и аккумулятором; по критериям: удовлетворения потребностей в энергии; стоимости системы; уровня загрязнения; не дают четкого ответа о предпочтительности той или иной системы по всем трем критериям [7] (Madziga, Rahil, Mansoor, 2018).

Приведенное выше возможное разнообразие проектов ЭО АП требует индивидуальной проработки каждого проекта на начальных стадиях ЖЦП, проведения научноисследовательских и опытно-конструкторских работ (НИОКР) с использованием моделирования потенциала предполагаемых к внедрению систем. Для ВИЭ учитываются также метеоусловия, рельеф и топология местности, что требует индивидуаль-

\section{ОБ АВTOPE:}

Жильцов Сергей Алексеевич, ассистент департамента инженерного бизнеса и менеджмента (zhiltsovsal mail.ru)

\section{ЦИТИРОВАТЬ СТАТЬЮ:}

Жильцов С.А. Методика реализации проекта автономного энергообеспечения в зависимости от стадий жизненного цикла // Вопросы инновационной экономики. - 2018. - Том 8. - № 4. - С. $731-740$. doi: 10.18334/vinec.8.4.39433 
ного подхода к оценке каждого проекта ЭО АП, прогнозирования потоков мощности, технико-экономической и социальной целесообразности, для эффективного обеспечения энергией регионов, расположенных вдали от мегаполисов и промышленных центров [8] (Kharlamova, Khalyasmaa, Eroshenko, 2017).

При использовании проектов гибридных микросетей также следует учитывать различия в жизненных циклах продуктов - компонентов систем. Если завершение всего проекта связывать с наступлением его полной окупаемости, то в прогнозировании ЖЦП следует учитывать, что ВИЭ имеют более высокие капитальные затраты на возведение, но низкие эксплуатационные расходы, в то время, как дизель-генераторы характеризуются обратной тенденцией и существенно меньшим временем жизни [9]. Соответственно, индивидуальные особенности каждого проекта ЭО АП требуют прогнозирования его жизненного цикла и выделения стадий ЖЦП для эффективной реализации проекта.

\section{Подходы к выделению стадий ЖЦП в сфере ЭО АП}

Анализ литературных данных относительно фаз, стадий, этапов ЖЦП позволил выявить разнородность представлений о стадиях проекта, выделяемых различными авторами. Базовые стадии ЖЦП, приведенные в трудах некоторых исследователей в области управления проектами, систематизированы на рисунке 1 .

Как было показано в предыдущем разделе статьи, проект ЭО АП разрабатывается индивидуально в зависимости от множества факторов микро- и макросреды, что обуславливает инновационный характер таких проектов и требует детальной проработки на начальной (концептуальной) фазе проекта (рuc. 2). В данном случае формулирование идеи, составление набора альтернативных проектов ЭО АП, выбор наилучшей альтернативы и утверждение концепции проекта, возможно, потребует проведения предварительных НИОКР на начальной стадии, выполняемых сторонними научными организациями, с привлечением маркетинговых и финансовых аналитиков для проведения ряда технико-экономических экспертиз. Такой предпроектный анализ в случае инновационного проекта требует проведения конкурсов (торгов) на выполнение предварительных исследований и экспертиз, которые следует учитывать в группе затрат по проекту.

Далее, на основе утвержденной концепции по результатам предпроектного анализа на начальной стадии организуется выполнение проектных работ, при этом проектировщиком может выступать независимая организация, не связанная с компанией, осуществляющей основную фазу реализации проекта, что также требует конкурсных процедур и учета затрат на соответствующих этапах ЖЦП. Кроме начальной фазы, представленные на рисунке 2 фазы проекта характерны как для инновационного, так и для традиционного проекта. Распределение уровня затрат и прибыли является условным, однако, следует учитывать, что для инновационных проектов с необходимостью НИОКР уровень затрат в начальной фазе может быть сопоставим с основными затратами в фазе реализации проекта. 
Луков В.А. выделяет фазы:

- начальная фаза (разработка концепции проекта, определение проекта, оценка альтернатив, апробация предложений, экспертиза, разработка и утверждение концепции);

- фаза разработки (разработка основных компонент проекта, формирование команды проекта, структурное планирование, организация и проведение торгов, заключение контрактов и субконтрактов, организация выполнения проектных работ);

- фаза реализации (выполнение основных работ проекта, необходимых для достижения основных целей проекта);

- завершающая фаза (достижение конечных целей проекта, подведение итогов, закрытие проекта).

Источник: Луков В.А. Социальное проектирование. - М.: Флинта, 2009.

Полковников А.В. с соавтором предлагает стадии:

- разработки концепции проекта;

- планирования проекта;

- его осуществления;

- завершения проекта.

Источник: Полковников А.В., Дубовик М.Ф. Управление проектами. Полный курс МВА. - М.: Олимп-Бизнес, 2012.

Борониной И.Н. со ссылкой на Мазура И.И. выделены этапы:

- технико-экономического обоснования;

- планирования и разработки проекта;

- производственный;

- заключительный.

Источник: Боронина И.Н., Сенук 3.В..

Основы управления проектами: уч. пособ. Екатеринбург, 2015.

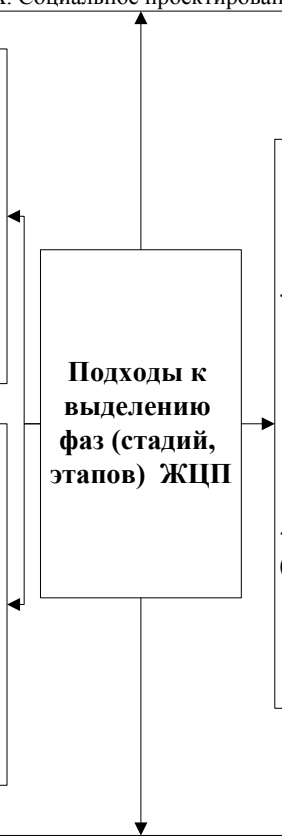

Туккель И.Л. с соавторами выделяет этапы:

- предпроектный анализ (анализ ситуации);

- формулировка концепции проекта (целеполагание);

- мобилизация ресурсов;

- реализация проекта (методы

достижения целей - управление проектами);

- мониторинг проекта;

- наращивание потенциала проекта

(перепланирование) или завершение (закрытие проекта).

Источник: Туккель И.Л., Сурина А.В.,

Культин Н.Б. Управление инновационными проектами. - СПб.: БХВ-Петербург, 2011.

Мазур И.И. с соавторами выделяют фазы:

- концептуальная фаза, включающая формулирование целей, анализ инвестиционных возможностей, обоснование осуществимости (технико-экономическое обоснование) и планирование проекта;

- фаза разработки проекта, включающая определение структуры работ и исполнителей, построение календарных графиков работ, бюджета проекта, разработку проектно-сметной документации, переговоры и заключение контрактов с подрядчиками и поставщиками;

- фаза выполнения проекта, включающая работы по его реализации (строительство, маркетинг, обучение персонала);

- фаза завершения проекта, включающая в общем случае приемочные испытания, опытную эксплуатацию и сдачу проекта в эксплуатацию;

- эксплуатационная фаза, включающая: приемку и запуск, замену оборудования, расширение, модернизацию, инновацию.

Источник: Мазур И.И., Шапиро В.Д., Ольдерогге Н.Г. Управление проектами: учебное пособие. - М.: Омега-Л, 2004.

Рисунок 1. Подходы к выделению фаз (стадий, этапов) ЖЦП

Источник: составлено автором 


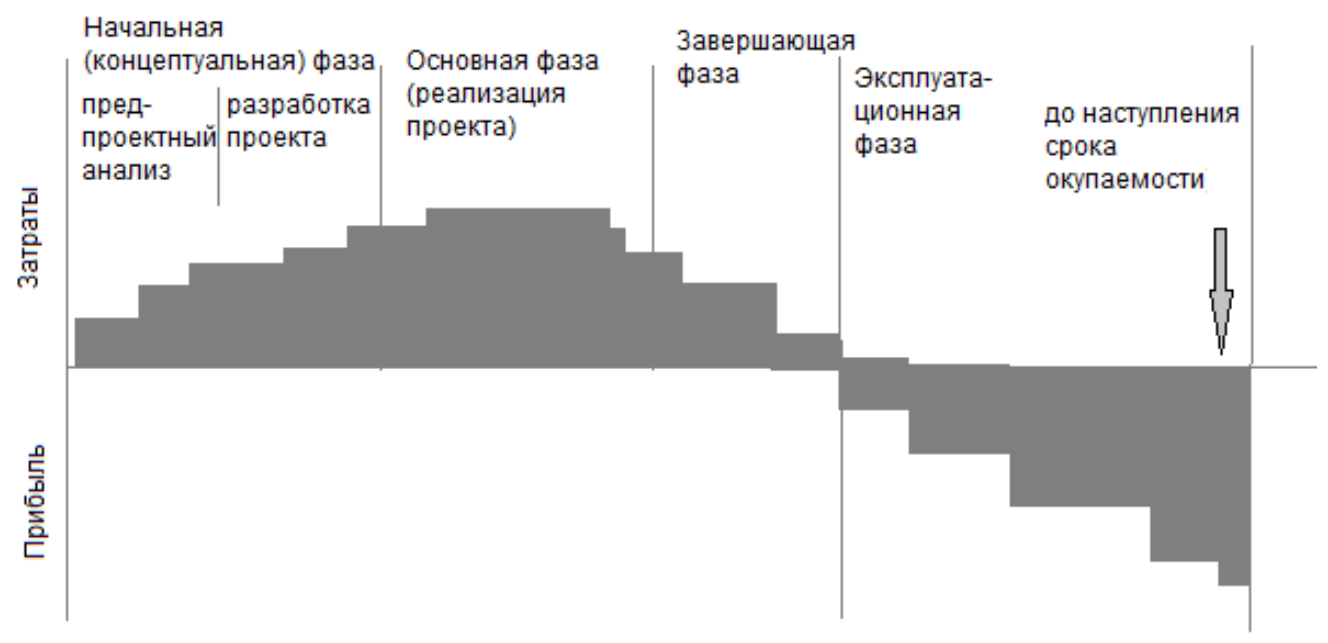

Рисунок 2. ЖЦП автономного энергообеспечения и условное распределение затрат и прибыли Источник: составлено автором

При выборе подхода рассмотрения ЖЦП, где эксплуатационная фаза и проект в целом завершается периодом его полной ликвидации, необходимо учитывать формирование прибыли после наступления срока окупаемости проекта, а также последующие затраты, связанные с его ликвидацией.

\section{Разработка методики реализации проекта ЭО АП в зависимости от стадий жизненного цикла}

Повышение эффективности реализации проекта большинство исследователей в области проектного менеджмента связывают с уточнением процессов и процедур, совершенствованием инструментов и методов, развитием компетенций и культуры, определением отчетности и ответственности. При этом именно способность снижения неопределенности на начальной стадии проекта приведет к лучшему пониманию проекта и, следовательно, положительно скажется на успешном управлении проектом и снижении рисков [10] (Mabelo, Sunjka, 2017).

Соответственно, в отличие от традиционных, легко структурируемых проектов, инновационный проект ЭО АП необходимо реализовывать на принципах системного проектирования, фокусируясь на определении потребностей клиентов и функциональных возможностей систем энергоснабжения в начале цикла разработки. Далее, после определения концепции проекта, следует документирование требований, операций, графика, стоимости, производительности, обучения и поддержки на последующих стадиях, а также мониторинга данных требований в течение ЖЦП в рамках ограничений затрат. 
В этой связи методика реализации проекта ЭО АП с усилением работ на начальной стадии заключается в следующем:

А. Начальная (концептуальная) фаза.

А.1. Этап предпроектного анализа.

A.1.1. Возникновение идеи проекта, формирование управленческой команды.

А.1.2. Определение альтернатив проекта.

А.1.2.1. Выполнение НИОКР для получения альтернативных технических решений ЭО АП.

A.1.2.2. Выполнение стратегических и маркетинговых исследований (PEST, SWOTанализ, потребители, конкуренты, контрагенты, эффективность ранее внедренных проектов ЭО АП) для формирования рыночных альтернатив.

А.1.2.3. Формирование портфеля альтернатив.

A.1.2.4. Технико-экономическое обоснование, финансовый и инвестиционный анализ, анализ рисков, коммерческой, социальной, бюджетной эффективности и пр.

При необходимости обращения к независимым научно-исследовательским, маркетинговым, финансовым и др. компаниям до выполнения пп. А.1.2.1-А.1.2.4 вводится работа А.1.2.0 - составление конкурсной документации, проведение торгов, заключение контрактов.

А.1.3. Утверждение альтернативы. Если по результатам раздела А.1. не установлено целесообразного к внедрению проекта ЭО АП, производится возврат к п. А.1.2.

А.2. Этап разработки проекта.

А.2.1 Уточнение целей и стратегии.

А.2.2. Структурирование работ (календарное, сетевое планирование).

А.2.3. Составление бюджетов и смет. Выявление объемов и источников собственного и внешнего финансирования, при необходимости кредитования или образования государственно-частных партнерств - составление бизнес-плана, инвестиционного меморандума и пр.

В случае образования разрыва во времени между этапами А.1. и А.2. следует повторить работу А.1.2.4.

А.2.4. Формирование команды проекта, в том числе контрагентов для работ, передаваемых на аутсортинг.

А.2.5 Утверждение порядка работ, контрольных, критических и целевых показателей проекта.

А.2.6. Выполнение непосредственно проектировочных работ. Если работы выполняются сторонней организацией - выявить объемы и источники финансирования, сформировать конкурсную документацию, провести торги, заключить договоры.

А.2.7. Утвердить проектную документацию. В случае необходимости, осуществить государственную экспертизу проектной документации. При необходимости скорректировать результаты п. А.1.2.4 и А.2.3 и повторить п. А.2.5 по данным п. А.2.6.

Б. Основная фаза (реализация проекта). 
Б.1. При необходимости возведения системы ЭО АП сторонними организациями проделать работы, аналогично А.2.6.

Б.2. Производить мониторинг реализации проекта по критическому пути, стоимости проекта, соответствия ресурсов, сметы, бюджета, рисков, качества и пр. в сравнении с плановыми показателями. Контроль за ходом проекта предлагается осуществлять с применением программных продуктов по управлению проектами, взаимоотношениями с контрагентами, жизненным циклом, расписаниями и т. д.

Б.3. Принятие корректирующих воздействий при обнаружении отклонений в п. Б.2. от утвержденных критериев в п. А.2.5.

В. Завершающая фаза.

В.1. Пуско-наладочные работы, приемка объекта, ввод системы ЭО АП в эксплуатацию.

В.2. Выявление отклонений в реализации проекта, не поддавшихся корректировке по п. Б.3, для последующего учета их в аналогичных проектах.

Г. Эксплуатационная фаза.

Г.1. Производить мониторинг работы системы, при необходимости осуществлять модернизацию, расширение функционала, цифровизацию и т. д.

Г.2. Систематизировать произведенные корректировки и изменения, а также затраты, связанные с ними, для учета в аналогичных проектах, если не были изначально предусмотрены в п. А.2.5.

В данной методике окончанием жизненного цикла проекта считается наступление его окупаемости, соответственно, до этого периода, работы раздела «Г» считаются продолжающимися. Предложенная методика позволяет гибко корректировать реализацию проекта на различных фазах ЖЦП, а также накапливать массив данных о происходящих отклонениях для повышения эффективности реализации последующих аналогичных проектов.

\section{Заключение}

Показана необходимость развития ЭО АП для повышения качества жизни и экономического развития удаленных территорий. Охарактеризованы возможные альтернативы проектов ЭО АП, в том числе с применением ВИЭ и гибридных систем, перспективы которых несомненны в связи с высокими выбросами парниковых газов от традиционных энергоресурсов и конечной природой ископаемых видов топлива. Выявлено, что каждый проект ЭО АП разрабатывается индивидуально с учетом географии местности, уровня потребности в энергии, ограничений в ресурсах, стоимости и др. факторов. Отсутствие типовых, легко тиражируемых решений приводит к необходимости разработки инновационных проектов, жизненный цикл которых имеет существенные отличия от традиционных проектов.

Систематизированы подходы к выделению фаз жизненного цикла и определено недостаточное структурирование начальной фазы проекта, являющейся ключевой в инновационных проектах с высокой неопределенностью. Предложена методика реа- 
лизации проекта ЭО АП на разных стадиях ЖЦП, позволяющая производить комплексную оценку альтернатив на начальной стадии проекта, а также гибко корректировать реализацию проекта на различных фазах ЖЦП и регистрировать отклонения для повышения эффективности реализации последующих аналогичных проектов.

\section{ИСТОЧнИКИ:}

1. Селиверстов В.Е. Региональное стратегическое планирование: от методологии к практике. - Новосибирск: Изд-во ИЭОПП СО РАН, 2013.

2. Кузнецов Е.Б. Национальный доклад об инновациях в России. - Москва, 2016.

3. Next-generation wind and solar power. - Paris: IEA Publications, 2016.

4. Ershov V. Economic Evaluation of Centralized and Autonomous Power Supply Systems in Oil and Gas Industry. - Prague: Master Thesis, 2017.

5. Fedak W., Ulbrich R., Anweiler S., Jarosz B. The Concept of Autonomous Power Supply System Fed with Renewable Energy Sources // Journal of Sustainable Development of Energy, Water and Environment Systems, 2017. - № 4.

6. Fikari S.G. Modeling and simulation of an autonomous hybrid power system, 2015.

7. Madziga M., Rahil A., Mansoor R. Comparison between three off-grid hybrid systems for electrification for Gwakwani Village // Environments, 2018. - № 57. - doi: 10.3390/ environments5050057.

8. Kharlamova N.V., Khalyasmaa A.I., Eroshenko S.A. Alternative power supply systems for remote industrial customers // IOP Conf. Series: Earth and Environmental Science, 2017. - № 72. - doi: 10.1088/1755-1315/72/1/012027.

9. Rural Electrification with Renewable Energy Technologies, quality standards and business models. Alliance for Rural Electrification. Brussels, 2011.

10. Mabelo P.B., Sunjka B.P. Application of systems engineering concepts to enhance project lifecycle methodologies // South African Journal of Industrial Engineering, 2017. - № 3.

\section{REFERENCES:}

Next-generation wind and solar power (2016). Paris: IEA Publications.

Ershov V. (2017). Economic Evaluation of Centralized and Autonomous Power Supply Systems in Oil and Gas Industry Prague: Master Thesis.

Fedak W., Ulbrich R., Anweiler S., Jarosz B. (2017). The Concept of Autonomous Power Supply System Fed with Renewable Energy Sources Journal of Sustainable Development of Energy, Water and Environment Systems. 5 (4).

Fikari S.G. (2015). Modeling and simulation of an autonomous hybrid power system Uppsala,

Kharlamova N.V., Khalyasmaa A.I., Eroshenko S.A. (2017). Alternative power supply systems for remote industrial customers IOP Conf. Series: Earth and Environmental Science. (72). doi: 10.1088/1755-1315/72/1/012027. 
Kuznetsov E.B. (2016). Natsionalnyy doklad ob innovatsiyakh v Rossii [National report on innovation in Russia] Moscow. (in Russian).

Mabelo P.B., Sunjka B.P. (2017). Application of systems engineering concepts to enhance project lifecycle methodologies South African Journal of Industrial Engineering. 28 (3).

Madziga M., Rahil A., Mansoor R. (2018). Comparison between three off-grid hybrid systems for electrification for Gwakwani Village Environments. 5 (57). doi: 10.3390/ environments5050057.

Seliverstov V.E. (2013). Regionalnoe strategicheskoe planirovanie: ot metodologii k praktike [Regional strategic planning: from methodology to practice] Novosibirsk: zd-vo IEOPP SO RAN. (in Russian). 